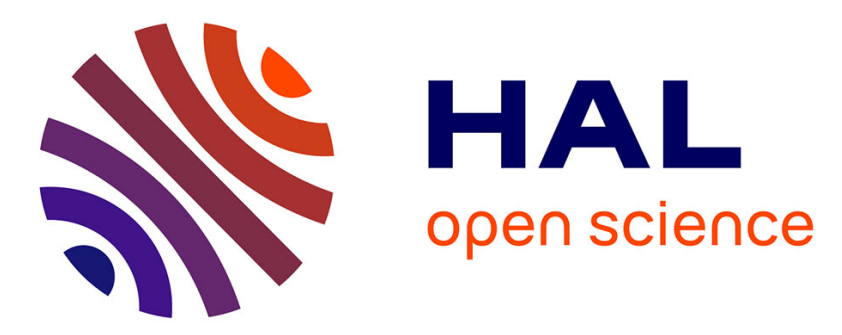

\title{
Model order reduction of quasi-static problems based on POD and PGD approaches
}

\author{
Thomas Henneron, Stéphane Clenet
}

\section{To cite this version:}

Thomas Henneron, Stéphane Clenet. Model order reduction of quasi-static problems based on POD and PGD approaches. European Physical Journal: Applied Physics, 2013, 5, pp.1-7. 10.1051/epjap/2013120411 . hal-00851978

\section{HAL Id: hal-00851978 \\ https://hal.science/hal-00851978}

Submitted on 2 Sep 2013

HAL is a multi-disciplinary open access archive for the deposit and dissemination of scientific research documents, whether they are published or not. The documents may come from teaching and research institutions in France or abroad, or from public or private research centers.
L'archive ouverte pluridisciplinaire HAL, est destinée au dépôt et à la diffusion de documents scientifiques de niveau recherche, publiés ou non, émanant des établissements d'enseignement et de recherche français ou étrangers, des laboratoires publics ou privés. 


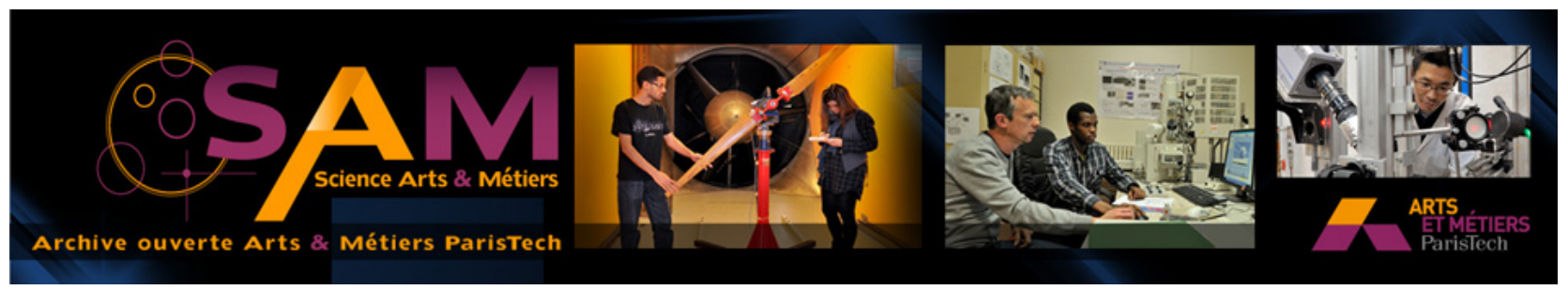

Science Arts \& Métiers (SAM)

is an open access repository that collects the work of Arts et Métiers ParisTech researchers and makes it freely available over the web where possible.

This is an author-deposited version published in: http://sam.ensam.eu

Handle ID: .http://hdl.handle.net/10985/7234

\section{To cite this version :}

Thomas HENNERON, Stéphane CLENET - Model order reduction of quasi-static problems based on POD and PGD approaches - The European Physical Journal Applied Physics n`5, p.1-7 2013 


\title{
Model order reduction of quasi-static problems based on POD and PGD approaches
}

\author{
Thomas Henneron $^{1}$ a and Stéphane Clénet $^{2}$ \\ 1 L2EP, Université Lille 1, 59655 Villeneuve dAscq, France \\ 2 L2EP, Arts et Métiers ParisTech, 59046 Lille, France
}

Received: date / Revised version: date

\begin{abstract}
In order to reduce the computation time of a quasi-static problem solved by the finite element method, methods of model order reduction can be applied. In this context, two approaches, the Proper Orthogonal Decomposition and the Proper Generalized Decomposition, are applied to the vector potential formulation used to solve the quasi-static problem. The developped methods are compared on an academic example.
\end{abstract}

\section{Introduction}

In quasi-static problems, the distributions of the magnetic and electric fields depend on time. By using a numerical approach, the Maxwell equations are discretized simultaneously in the space and the time domains. To solve this kind of problem, the finite element method is commonly used to approximate the distribution of the fields in the space domain. The time domain can be discretised by an implicit or explicit Euler scheme or any time-stepping schemes. If the mesh of the space domain is fine and a small time step is used, the computation time can be significant. To tackle this issue, an alternative is to use model order reduction methods. These approaches consist in expanding the solution of the initial problem in a reduced basis. In the litterature, several approaches have been developed, we can distinguish the $a$-priori and a-posteriori methods. In this work, two methods of model order reduction are applied to solve quasi-static field problems. The first method is the Proper Orthogonal Decomposition (POD) approach which is an a-posteriori method [1]. It consists in performing a projection onto the reduced basis of the space domain. In the discrete domain, the Snapshot approach is mainly used to determine the discrete projection operator [2]. In computational electromagnetics, the POD method has been applied, for example, to study the behavior of magnetic core with non linear static hysteresis [3] or to solve an electroquasistatic field problem [4]. The second approach presented is the Proper Generalized Decomposition (PGD) method which is an a-priori method. This is based on the separated representation of the solution, as for example, in function of time and space [5][6][7]. In computational electromagnetics, the PGD method has been applied to study a fuel cell polymeric membrane model [8], the skin effect in a conducting domain [9][10]

\footnotetext{
a Present address: thomas.henneron@univ-lille1.fr
}

or a soft magnetic composite microstructure [11]. These two methods of model order reduction are investigated to solve a quasi-static problem using the vector potential formulation. In a first part, the quasi-static problem and the vector potential formulation are presented. In the second part, the POD and PGD approaches are developped. Finally, a 3D academic example is studied with both methods. The results are compared with those obtained by a more classical approach, fully discretised in the time and space domains.

\section{Maxwell's equations and vector potential formulation}

Let us consider a domain $D$ of boundary $\Gamma\left(\Gamma=\Gamma_{B} \cup \Gamma_{H}\right.$ and $\left.\Gamma_{B} \cap \Gamma_{H}=\emptyset\right)$. In $D$, a conducting domain $D_{c}$ of boundary $\Gamma_{c}\left(\Gamma_{c}=\Gamma_{\text {Jind }} \cup \Gamma_{E}\right.$ and $\left.\Gamma_{E} \subset \Gamma_{B}\right)$ is introduced (Fig. 1).

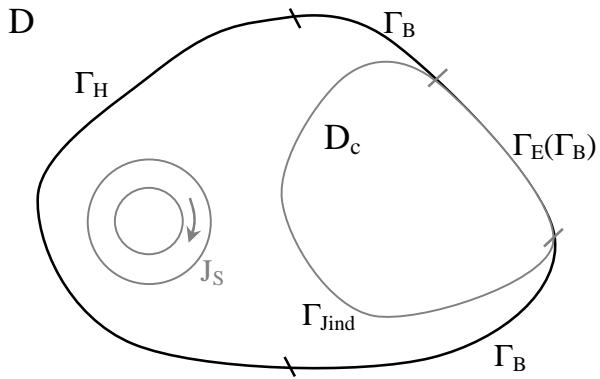

Fig. 1. Computational domain 
The quasi-static field problem can be represented by Maxwell's equations without the displacement current,

$$
\begin{aligned}
& \operatorname{curl} \boldsymbol{E}(\boldsymbol{x}, t)=-\partial_{t} \boldsymbol{B}(\boldsymbol{x}, t), \\
& \operatorname{curl} \boldsymbol{H}(\boldsymbol{x}, t)=\boldsymbol{J}_{\text {ind }}(\boldsymbol{x}, t)+\boldsymbol{J}_{s}(\boldsymbol{x}, t), \\
& \operatorname{div} \boldsymbol{B}(\boldsymbol{x}, t)=0, \\
& \operatorname{div}\left(\boldsymbol{J}_{\text {ind }}(\boldsymbol{x}, t)+\boldsymbol{J}_{s}(\boldsymbol{x}, t)\right)=0
\end{aligned}
$$

with $\boldsymbol{B}$ the magnetic flux density, $\boldsymbol{H}$ the magnetic field, $\boldsymbol{E}$ the electric field, $\boldsymbol{J}_{s}$ the known current density flowing through the stranded inductors and $\boldsymbol{J}_{\text {ind }}$ the eddy current density in the conducting domain. To take into account the material behavior, the constitutive relations between the fields $\boldsymbol{B}$ and $\boldsymbol{H}$ and the fields $\boldsymbol{J}_{\text {ind }}$ and $\boldsymbol{E}$ must be considered. In the linear case, we have

$$
\begin{aligned}
& \boldsymbol{B}(\boldsymbol{x}, t)=\mu_{0} \mu_{r} \boldsymbol{H}(\boldsymbol{x}, t), \\
& \boldsymbol{J}_{\text {ind }}(\boldsymbol{x}, t)=\sigma \boldsymbol{E}(\boldsymbol{x}, t)
\end{aligned}
$$

with $\mu_{0}$ the magnetic permeability of the vacuum, $\mu_{r}$ the relative permeability and $\sigma$ the electric conductivity. To impose the unicity of the solution, boundary conditions must be added

$$
\begin{aligned}
& \boldsymbol{H}(\boldsymbol{x}, t) \times \boldsymbol{n}=0 \quad \text { on } \quad \Gamma_{H}, \\
& \boldsymbol{B}(\boldsymbol{x}, t) \cdot \boldsymbol{n}=0 \quad \text { on } \quad \Gamma_{B}, \\
& \boldsymbol{E}(\boldsymbol{x}, t) \times \boldsymbol{n}=0 \quad \text { on } \quad \Gamma_{E}, \\
& \boldsymbol{J}_{\text {ind }}(\boldsymbol{x}, t) \cdot \boldsymbol{n}=0 \quad \text { on } \quad \Gamma_{\text {Jind }}
\end{aligned}
$$

with $\boldsymbol{n}$ the outward unit normal vector.

To solve the previous problem, the $\boldsymbol{A}_{*}$ formulation can be used. A magnetic vector potential $\boldsymbol{A}_{*}(\boldsymbol{x}, t)$ is defined in the whole domain by using (1) and (3)

$$
\begin{aligned}
& \boldsymbol{B}(\boldsymbol{x}, t)=\operatorname{curl} \boldsymbol{A}_{*}(\boldsymbol{x}, t), \boldsymbol{E}(\boldsymbol{x}, t)=-\partial_{t} \boldsymbol{A}_{*}(\boldsymbol{x}, t)(11) \\
& \text { and } \quad \boldsymbol{A}_{*}(\boldsymbol{x}, t) \times \boldsymbol{n}=0 \quad \text { on } \quad \Gamma_{B} .
\end{aligned}
$$

Combining the previous relations and the behavior laws in (2), we obtain the vector potential formulation of the problem which must be solved in $D \times[0, T]$ with $T$ the length of the time interval. The weak form to be solved is then written

$$
\begin{aligned}
& \int_{0}^{T} \int_{D} \frac{1}{\mu} \operatorname{curl} \boldsymbol{A}_{*}(\boldsymbol{x}, t) \cdot \operatorname{curl} \boldsymbol{A}^{\prime}(\boldsymbol{x}) \\
& +\sigma \partial_{t} \boldsymbol{A}_{*}(\boldsymbol{x}, t) \cdot \boldsymbol{A}^{\prime}(\boldsymbol{x}) d D d t \\
& =\int_{0}^{T} \int_{D} \boldsymbol{J}_{s}(\boldsymbol{x}, t) \cdot \boldsymbol{A}^{\prime}(\boldsymbol{x}) d D d t
\end{aligned}
$$

with $\boldsymbol{A}^{\prime}(\boldsymbol{x})$ a test function defined on the same space as $\boldsymbol{A}_{*}$. In the $3 \mathrm{D}$ case, the potential $\boldsymbol{A}_{*}(\boldsymbol{x}, t)$ is semidiscretised in space by using the edge elements:

$$
\boldsymbol{A}_{*}(\boldsymbol{x}, t)=\sum_{i=1}^{N_{e}} A_{i}(t) \boldsymbol{w}_{a i}(\boldsymbol{x})
$$

with $\boldsymbol{w}_{a i}$ the edge shape function associated with the $i$-th edge, $A_{i}(t)$ a function of time and $N_{e}$ the number of edges. We denote by $\boldsymbol{A}_{D}(t)$ the vector of components $\left(A_{i}(t)\right)_{1 \leq i \leq N_{e}}$. Then a system of coupled ordinary differential equations has to be solved

$$
\begin{aligned}
& \boldsymbol{M} \boldsymbol{A}_{D}(t)+\boldsymbol{N} \frac{d \boldsymbol{A}_{D}(t)}{d t}=\boldsymbol{F}(t) \\
& \text { with } \quad M_{i, j}=\int_{D} \frac{1}{\mu} \operatorname{curl} \boldsymbol{w}_{a i}(\boldsymbol{x}) \cdot \operatorname{curl} \boldsymbol{w}_{a j}(\boldsymbol{x}) d D \\
& N_{i, j}=\int_{D_{c}} \sigma \boldsymbol{w}_{a i}(\boldsymbol{x}) \cdot \boldsymbol{w}_{a j}(\boldsymbol{x}) d D_{c} \\
& F_{i}(t)=\int_{D} \boldsymbol{J}_{s}(\boldsymbol{x}, t) \cdot \boldsymbol{w}_{a i}(\boldsymbol{x}) d D
\end{aligned}
$$

Generally, to solve (14) in the time domain, an Euler scheme is used. In the following, the known current density $\boldsymbol{J}_{s}$ in the stranded inductors is expressed by a separated representation

$$
\boldsymbol{J}_{s}(\boldsymbol{x}, t)=\boldsymbol{N}(\boldsymbol{x}) i(t)
$$

with $\boldsymbol{N}(\boldsymbol{x})$ the source field and $i(t)$ the evolution of the current versus the time that is supposed to be known.

\section{Model order reduction}

The Proper Orthogonal Decomposition and the Proper Generalized Decomposition methods are based on a separated representation of space and time functions of the potential $\boldsymbol{A}_{*}(\boldsymbol{x}, t)$

$$
\boldsymbol{A}_{*}(\boldsymbol{x}, t) \approx \sum_{n=1}^{M} \boldsymbol{R}_{n}(\boldsymbol{x}) S_{n}(t)
$$

with $\boldsymbol{R}_{n}(\boldsymbol{x})$ defined on $D, S_{n}(t)$ defined on $[0, T]$ and $M$ the number of modes taken into account for the approximation of $\boldsymbol{A}_{*}(\boldsymbol{x}, t)$. The aim of the POD and PGD methods is to find the "best" separated representation with M functions.

\subsection{Proper Orthogonal Decomposition}

With the POD method [1], we consider that the functions $\boldsymbol{R}_{n}(\boldsymbol{x})$ are an orthogonal basis, that is

$$
\int_{D} \boldsymbol{R}_{i}(\boldsymbol{x}) \cdot \boldsymbol{R}_{j}(\boldsymbol{x}) d D=0 \quad \text { if } \quad i \neq j .
$$

The functions $S_{n}(t)$ can be expressed from the projection of $\boldsymbol{A}_{*}(\boldsymbol{x}, t)$ on the basis of functions $\boldsymbol{R}_{n}(\boldsymbol{x})$ such that

$$
S_{n}(t)=\int_{D} \boldsymbol{A}_{*}(\boldsymbol{x}, t) \cdot \boldsymbol{R}_{n}(\boldsymbol{x}) d D .
$$

To determine the set of functions $\boldsymbol{R}_{n}(\boldsymbol{x})$, we aim at minimizing the quantity

$$
\begin{aligned}
& \left\|\boldsymbol{A}_{*}(\boldsymbol{x}, t)-\sum_{n=1}^{M} \boldsymbol{R}_{n}(\boldsymbol{x}) S_{n}(t)\right\|^{2} \\
& =\left\|\boldsymbol{A}_{*}(\boldsymbol{x}, t)-\sum_{n=1}^{M}\left(\int_{D} \boldsymbol{A}_{*}(\boldsymbol{x}, t) \cdot \boldsymbol{R}_{n}(\boldsymbol{x}) d D\right) \boldsymbol{R}_{n}(\boldsymbol{x})\right\|^{2}
\end{aligned}
$$


To determine a discrete representation of the functions $\boldsymbol{R}_{n}(\boldsymbol{x})$, the Snapshot method can be used [2]. In a first step, the system (14) is solved for the first $M$ time steps (called Snapshots). The $M$ vectors of $\boldsymbol{A}_{D}^{i}$ obtained at each time step are gathered in a matrix $\boldsymbol{A}_{S}$ of size $N_{e} \times M$. In the discrete domain, (18) and (19) can be written

$$
\begin{aligned}
& \boldsymbol{P}=\boldsymbol{A}_{S} \boldsymbol{R}, \\
& \boldsymbol{A}_{S}=\boldsymbol{P} \boldsymbol{A}_{S r}
\end{aligned}
$$

with $\boldsymbol{A}_{S r}$ the matrix of the Snapshot in the reduced basis, $\boldsymbol{R}$ a matrix whose column $i$ corresponds to the discrete representation of the function $R_{i}, \boldsymbol{P}^{t}$ the discrete projection operator between the values of $\boldsymbol{A}_{*}$ in the basis of the $N_{e}$ edge functions and the reduced basis. In order to determine the expression of $\boldsymbol{P}$, a Singular Value Decomposition (SVD) is applied to the matrix of Snapshots such that $\boldsymbol{A}_{S}=\boldsymbol{V} \boldsymbol{\Sigma} \boldsymbol{W}^{t}$ with $\boldsymbol{\Sigma}$ the diagonal matrix of the singular values, $\boldsymbol{V}$ and $\boldsymbol{W}$ the orthogonal matrices of the left and right singular vectors. By combining (20), (21) and the SVD of $\boldsymbol{A}_{S}$, we have

$$
\boldsymbol{A}_{S}=\boldsymbol{V} \boldsymbol{\Sigma} \boldsymbol{W}^{t} \boldsymbol{R} \boldsymbol{A}_{S r} .
$$

By fixing $\boldsymbol{R}=\boldsymbol{W}$, since $\boldsymbol{W}$ is orthogonal $\boldsymbol{W} \boldsymbol{W}^{t}=\boldsymbol{I}$, the previous equation can be simplified such that

$$
\boldsymbol{A}_{S}=\boldsymbol{V} \boldsymbol{\Sigma} \boldsymbol{A}_{S r}
$$

By identification, the expression of $\boldsymbol{P}$ can be defined such that $\boldsymbol{P}=\boldsymbol{V} \boldsymbol{\Sigma}$. Another approach to obtain the expression of $\boldsymbol{P}$ can be carried out by the calculation of the SVD of the correlation matrix defined by $\boldsymbol{A}_{S}^{t} \boldsymbol{A}_{S}$. The reduced matrix system can be deduced by using the operator $\boldsymbol{P}$ in (14) to obtain

$$
\begin{aligned}
& \boldsymbol{M}_{r} \boldsymbol{A}_{r}(t)+\boldsymbol{N}_{r} \frac{d \boldsymbol{A}_{r}(t)}{d t}=\boldsymbol{F}_{r}(t) \\
& \text { with } \quad \boldsymbol{M}_{r}=\boldsymbol{P}^{t} \boldsymbol{M} \boldsymbol{P}, \quad \boldsymbol{N}_{r}=\boldsymbol{P}^{t} \boldsymbol{N} \boldsymbol{P} \\
& \text { and } \quad \boldsymbol{F}_{r}(t)=\boldsymbol{P}^{t} \boldsymbol{F}(t) .
\end{aligned}
$$

The size of the matrices $\boldsymbol{M}_{r}$ and $\boldsymbol{N}_{r}$ and the vectors $\boldsymbol{F}_{r}$ and $\boldsymbol{A}_{r}$ depend on the number of Snapshots that is generally much smaller than the number of edges of the mesh. To obtain the solution on $[0, T]$, the reduced matrix system is solved all time steps. For each time step, the solution on the original mesh $\boldsymbol{A}_{D}(t)$ can be retrieved from the solution $\boldsymbol{A}_{r}(t)$ of the reduced model by

$$
\boldsymbol{A}_{D}(t)=\boldsymbol{P}^{t} \boldsymbol{A}_{r}(t) .
$$

\subsection{Proper Generalized Decomposition}

With the PGD approach, the weak formulation defined by (12) is considered. To compute the functions $\boldsymbol{R}_{n}(\boldsymbol{x})$ and $S_{n}(t)$, an iterative enrichment method is used. The couple $\left(\boldsymbol{R}_{n}(\boldsymbol{x}), S_{n}(t)\right)$ is calculated regarding the previous couples $\left(\boldsymbol{R}_{i}(\boldsymbol{x}), S_{i}(t)\right)$ with $i \in[1, n-1]$. In this case, the test function $\boldsymbol{A}^{\prime}$ can be written such that

$$
\boldsymbol{A}^{\prime}(\boldsymbol{x}, t)=\boldsymbol{R}_{n}^{\prime}(\boldsymbol{x}) S_{n}(t)+\boldsymbol{R}_{n}(\boldsymbol{x}) S_{n}^{\prime}(t)
$$

with $\boldsymbol{R}_{n}^{\prime}(\boldsymbol{x})$ and $S_{n}^{\prime}(t)$ the test functions defined in the same spaces as $\boldsymbol{R}_{n}(\boldsymbol{x})$ and $S_{n}(t)$ respectively. Each couple $\left(\boldsymbol{R}_{n}(\boldsymbol{x}), S_{n}(t)\right)$ is calculated by solving iteratively two equations determined from (12). First, we suppose that $\boldsymbol{R}_{n}(\boldsymbol{x})$ is known. Then, the function $\boldsymbol{R}_{n}^{\prime}(\boldsymbol{x})$ vanishes in (26) and the test function $\boldsymbol{A}^{\prime}$ is equal to $\boldsymbol{R}_{n}(\boldsymbol{x}) S_{n}^{\prime}(t)$. Equation (12) is solved in order to determine the function $S_{n}(t)$. This can be rewritten

$$
\begin{aligned}
& \int_{D} \frac{1}{\mu} \operatorname{curl}_{n}(\boldsymbol{x}) \cdot \operatorname{curl} \boldsymbol{R}_{n}(\boldsymbol{x}) d D \int_{0}^{T} S_{n}(t) \cdot S_{n}^{\prime}(t) d t \\
& +\int_{D} \sigma \boldsymbol{R}_{n}(\boldsymbol{x}) \cdot \boldsymbol{R}_{n}(\boldsymbol{x}) d D \int_{0}^{T} \partial_{t} S_{n}(t) \cdot S_{n}^{\prime}(t) d t \\
& =\int_{D} \boldsymbol{N}(\boldsymbol{x}) \cdot \boldsymbol{R}_{n}(\boldsymbol{x}) d D \int_{0}^{T} i(t) \cdot S_{n}^{\prime}(t) d t \\
& -\sum_{i=1}^{n-1} \int_{D} \frac{1}{\mu} \operatorname{curl} \boldsymbol{R}_{i}(\boldsymbol{x}) \cdot \operatorname{curl} \boldsymbol{R}_{n}(\boldsymbol{x}) d D \int_{0}^{T} S_{i}(t) \cdot S_{n}^{\prime}(t) d t \\
& -\sum_{i=1}^{n-1} \int_{D} \sigma \boldsymbol{R}_{i}(\boldsymbol{x}) \cdot \boldsymbol{R}_{n}(\boldsymbol{x}) d D \int_{0}^{T} \partial_{t} S_{i}(t) \cdot S_{n}^{\prime}(t) d t . \quad(27)
\end{aligned}
$$

One can note that the previous equation is a weak form of the following Ordinary Differential Equation

$$
\begin{aligned}
& A_{R} S_{n}(t)+B_{R} \frac{d S_{n}(t)}{d t}=C_{S}(t) \\
& A_{R}=\int_{D} \frac{1}{\mu} \operatorname{curl} \boldsymbol{R}_{n}(\boldsymbol{x}) \cdot \operatorname{curl} \boldsymbol{R}_{n}(\boldsymbol{x}) d D \\
& B_{R}=\int_{D} \sigma \boldsymbol{R}_{n}(\boldsymbol{x}) \cdot \boldsymbol{R}_{n}(\boldsymbol{x}) d D \\
& C_{S}(t)=i(t) \int_{D} \boldsymbol{N}(\boldsymbol{x}) \cdot \boldsymbol{R}_{n}(\boldsymbol{x}) d D \\
& -\sum_{i=1}^{n-1} S_{i}(t) \int_{D} \frac{1}{\mu} \operatorname{curl} \boldsymbol{R}_{i}(\boldsymbol{x}) \cdot \operatorname{curl} \boldsymbol{R}_{n}(\boldsymbol{x}) d D \\
& -\sum_{i=1}^{n-1} \frac{d S_{i}(t)}{d t} \int_{D} \sigma \boldsymbol{R}_{i}(\boldsymbol{x}) \cdot \boldsymbol{R}_{n}(\boldsymbol{x}) d D .
\end{aligned}
$$

Secondly, we compute the function $\boldsymbol{R}_{n}(\boldsymbol{x})$ assuming that $S_{n}(t)$ is known. In this case, the function $S_{n}^{\prime}(t)$ vanishes in (26) and the test function $\boldsymbol{A}^{\prime}$ is equal to $\boldsymbol{R}_{n}^{\prime}(\boldsymbol{x}) S_{n}(t)$. To determine $\boldsymbol{R}_{n}(\boldsymbol{x})$, the relation (12) is solved with these conditions. This corresponds to

$$
\begin{aligned}
& \int_{0}^{T} S_{n}(t) \cdot S_{n}(t) d t \int_{D} \frac{1}{\mu} \operatorname{curl} \boldsymbol{R}_{n}(\boldsymbol{x}) \cdot \mathbf{c u r l} \boldsymbol{R}_{n}^{\prime}(\boldsymbol{x}) d D \\
& +\int_{0}^{T} \partial_{t} S_{n}(t) \cdot S_{n}(t) d t \int_{D} \sigma \boldsymbol{R}_{n}(\boldsymbol{x}) \cdot \boldsymbol{R}_{n}^{\prime}(\boldsymbol{x}) d D \\
& =\int_{0}^{T} i(t) \cdot S_{n}(t) d t \int_{D} \boldsymbol{N}(\boldsymbol{x}) \cdot \boldsymbol{R}_{n}^{\prime}(\boldsymbol{x}) d D \\
& -\sum_{i=1}^{n-1} \int_{0}^{T} S_{i}(t) \cdot S_{n}(t) d t \int_{D} \frac{1}{\mu} \operatorname{curl} \boldsymbol{R}_{i}(\boldsymbol{x}) \cdot \mathbf{c u r l} \boldsymbol{R}_{n}^{\prime}(\boldsymbol{x}) d D \\
& -\sum_{i=1}^{n-1} \int_{0}^{T} \partial_{t} S_{i}(t) \cdot S_{n}(t) \int_{D} \sigma \boldsymbol{R}_{i}(\boldsymbol{x}) \cdot \boldsymbol{R}_{n}^{\prime}(\boldsymbol{x}) d D d t .
\end{aligned}
$$


The equation is also a weak form of a Partial Derivative Equation that can be written under the form

$$
\begin{aligned}
& A_{S}\left(\frac{1}{\mu} \mathbf{R}_{n}(\boldsymbol{x})\right)+B_{S} \sigma \boldsymbol{R}_{n}(\boldsymbol{x})=C_{R}(\boldsymbol{x}) \\
& A_{S}=\int_{0}^{T} S_{n}(t) \cdot S_{n}(t) d t \\
& B_{S}=\int_{0}^{T} \partial_{t} S_{n}(t) \cdot S_{n}(t) d t \\
& C_{R}(\boldsymbol{x})=\boldsymbol{N}(\boldsymbol{x}) \int_{0}^{T} i(t) \cdot S_{n}(t) d t \\
& -\sum_{i=1}^{n-1} \operatorname{curl}\left(\frac{1}{\mu} \operatorname{curl} \boldsymbol{R}_{i}(\boldsymbol{x})\right) \int_{0}^{T} S_{i}(t) \cdot S_{n}(t) d t \\
& -\sum_{i=1}^{n-1} \sigma \boldsymbol{R}_{i}(\boldsymbol{x}) \int_{0}^{T} \partial_{t} S_{i}(t) \cdot S_{n}(t) d t .
\end{aligned}
$$

The solution $\left(\boldsymbol{R}_{n}(\boldsymbol{x}), S_{n}(t)\right)$ verifies the ODE (28) and the PDE (30). This solution is obtained by an iterative procedure. In our case, we assure that $S_{n}(t)^{0}=1$ on $[0, T]$, then solving (30) we obtain $\boldsymbol{R}_{n}(\boldsymbol{x})^{0}$. Solving (28) taking $\boldsymbol{R}_{n}(\boldsymbol{x})$ $=\boldsymbol{R}_{n}(\boldsymbol{x})^{0}$, we obtain $S_{n}(t)^{1}$ and then the equation (28) is solved with $S_{n}(t)=S_{n}(t)^{1}$ and so on. This procedure is stopped once the difference between two consecutive iterates is sufficiently small. If we denote by $\left(\boldsymbol{R}_{n}(\boldsymbol{x})^{j}, S_{n}(t)^{j}\right)$ and $\left(\boldsymbol{R}_{n}(\boldsymbol{x})^{j-1}, S_{n}(t)^{j-1}\right)$ the couples of functions determined at the iteration $j$ and $j-1$.

The convergence proof of the separated solution representation methods has been given in [12]. Our problem does not belong to this class of problem. However, even though the proof is not given, our problem is similar to other ones which have been solved with the PGD approach and for which no convergence proof has been given [13]. In term of solution, the ODE formulation (28) can be easily solved by the Euler implicit scheme. The functions $S_{n}(t)$ and $i(t)$ are discretised in each time step. The weak formulation (29) is solved to determine the functions $\boldsymbol{R}_{n}(\boldsymbol{x})$, for example, by using the finite element method. The functions $\boldsymbol{R}_{n}(\boldsymbol{x})$ and $\boldsymbol{N}(\boldsymbol{x})$ are discretised in the edge shape function space with the same boundary conditions as $\boldsymbol{A}_{*}$ and in the facet shape function space respectively [14].

\section{Application}

As a 3D application example, a stranded inductor located between two conducting plates is considered. Due to the symmetries of the device, only one eighth of the system has been modeled (Fig. 2). The inductor is supplied by a sinusoidal current with a magnitude equal to $1 \mathrm{~A}$ and a frequency of $20 \mathrm{kHz}$. The number of turns of the inductor is equal to 100 . The relative magnetic permeability of the conducting plate is fixed to 1 and its electrical conductivity to $10 \mathrm{kS} / \mathrm{m}$. The 3D spatial mesh has 14970 nodes and 80199 tetrahedrons. The problem has been solved using the POD and PGD approaches applied to the $\boldsymbol{A}_{*}$ formulation. In order to evaluate the efficiency of the proposed models, the $\boldsymbol{A}_{*}$ formulation has been solved using a time stepping Finite Element Method. This approach will be considered in the following as the reference.

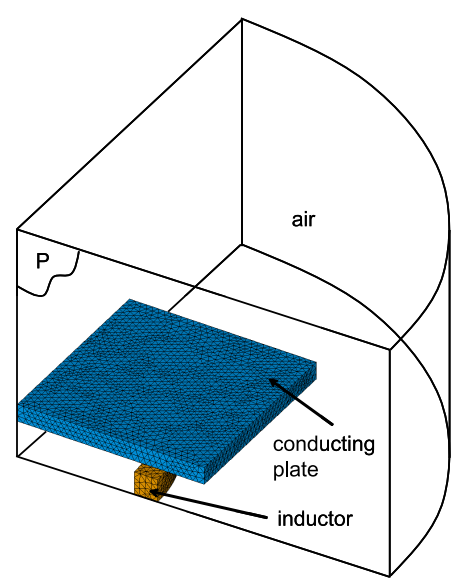

Fig. 2. Example of application

In the following, we denote by MPOD, MPGD and MREF the models obtained from the POD, PGD and classic approaches.

\subsection{Influence of the number of modes on the global values}

The length of the time interval is fixed to $0.1 \mathrm{~ms}$ with 60 time steps. In order to evaluate the influence of the number of modes $\mathrm{M}$ on the global values, the evolutions of Joule losses versus the time obtained from MPOD and MPGD are compared with those computed from MREF in Fig. (3) and (4) respectively. We can observe that the number of modes influences the evolution of the Joule losses for both methods. To estimate the convergence versus the number of modes of these curves, an error estimator $\epsilon_{(P j)}$ is defined such that

$$
\epsilon_{(P j)}=\frac{\left\|P j_{r e f}-P j_{e s t}\right\|_{2}}{\left\|P j_{r e f}\right\|_{2}}
$$

with $P j_{r e f}$ and $P j_{e s t}$ the vectors of Joule losses for all time steps obtained from MREF and MPOD or MPGD. Figure (5) presents the evolution of $\epsilon_{(P j)}$ versus the number of modes. The curves of error converge until zero. With MPOD the convergence is faster than with the MPGD. To obtain an error inferior to 0.1 percent, 5 modes are required with the MPOD and 12 with the MPGD.

In the case of MPGD, the evolutions of the functions $S_{n}(t)$ in the time domain are determined. In figure (6), the evolutions of the functions $S_{n}(t)$ for the four first modes are presented. A transient state can be observed on all curves. This transient state can also be observed on the evolution of the Joule Losses (Fig. (4)). 


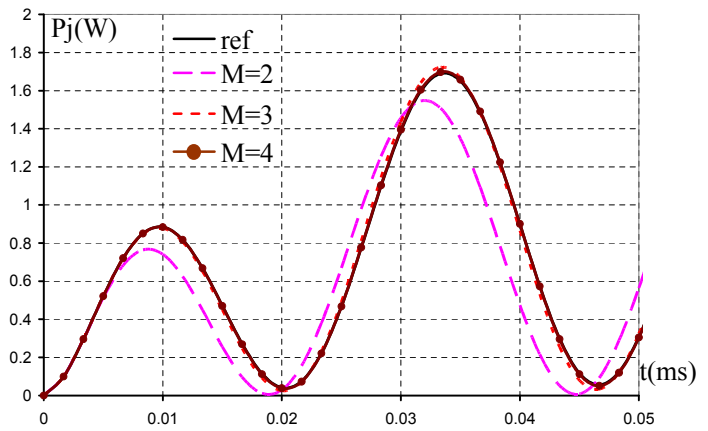

Fig. 3. Joules losses obtained from the POD method

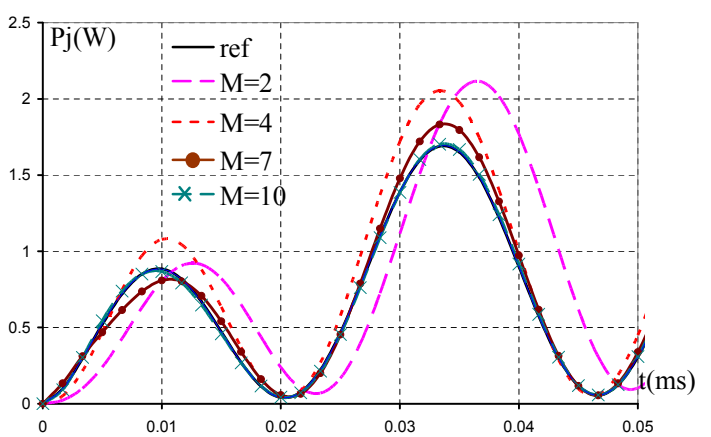

Fig. 4. Joules losses obtained from the PGD method

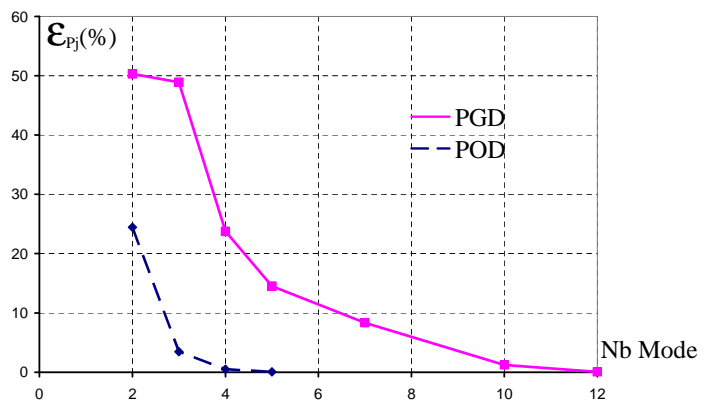

Fig. 5. Error on the Joules losses versus the number of modes for both methods

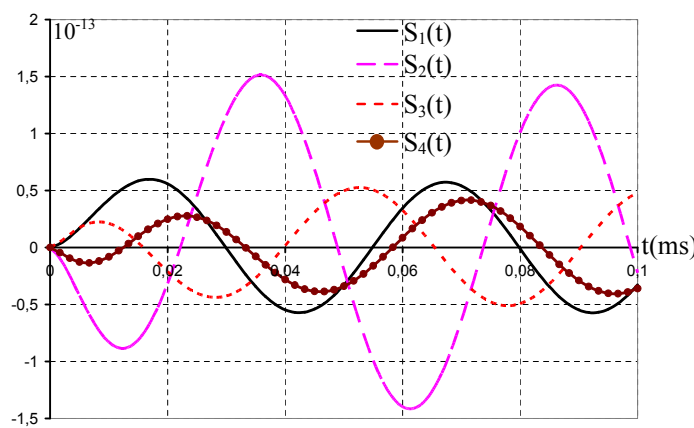

Fig. 6. Evolution of the four first modes $S_{n}(t)$

\subsection{Influence of the number of modes on the fields distribution}

As the vector potential is approximated by a sum of contribution associated with each mode, it is possible to give a distribution of the magnetic flux density and of the eddy current density for each mode. For MPGD, the figures (7) and (8) present the distribution of $\boldsymbol{B}$ on a section $\mathrm{P}$ presented in Fig. (2) for the first and the second mode. For the POD approach, the figures (9) and (10) present the distributions of $\boldsymbol{B}$ for the first and second mode.

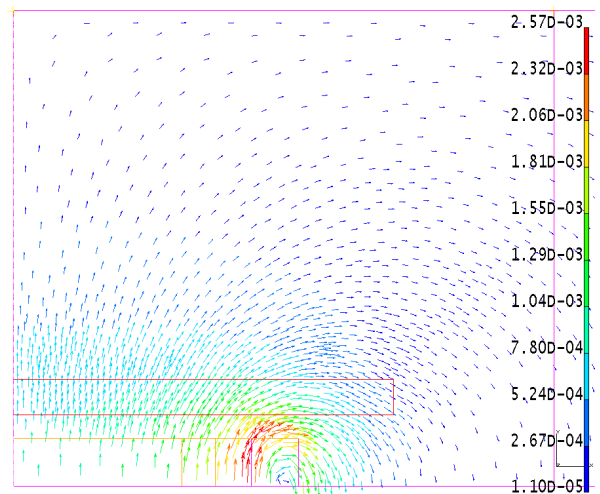

Fig. 7. Distribution of $\boldsymbol{B}(T)$ for the first mode given by MPGD

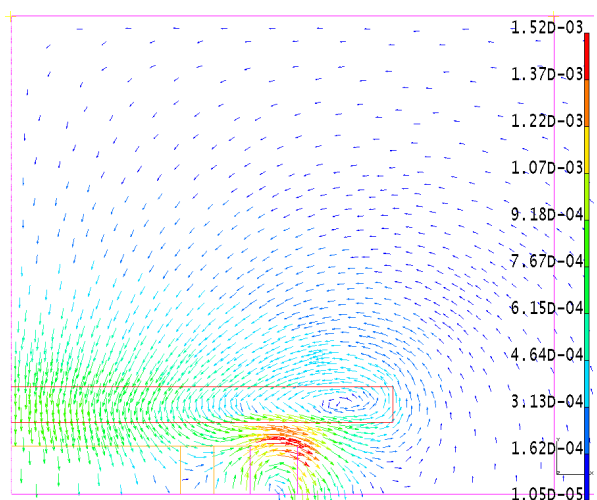

Fig. 8. Distribution of $\boldsymbol{B}(T)$ for the second mode given by MPGD

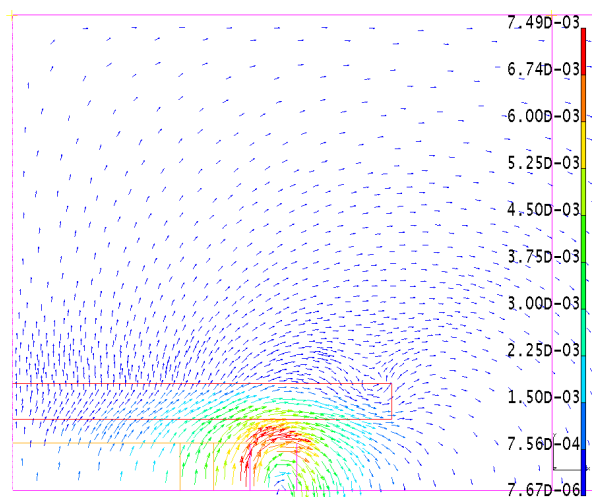

Fig. 9. Distribution of $\boldsymbol{B}(T)$ for the first mode given by MPOD 


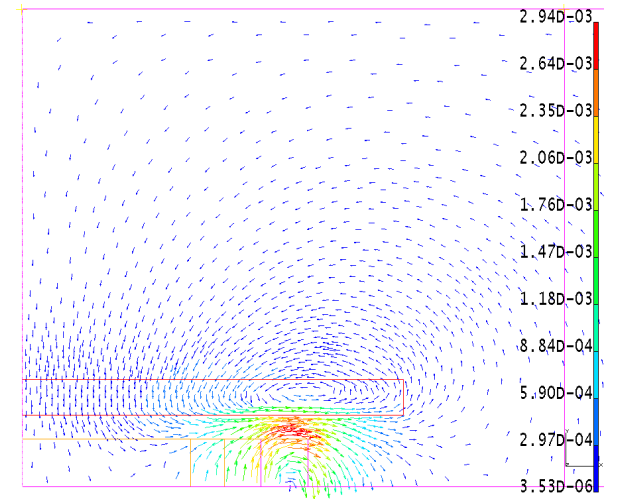

Fig. 10. Distribution of $\boldsymbol{B}(T)$ for the second mode given by MPOD

Since the reduced basis are differents, the distributions of $\boldsymbol{B}$ obtained from MPOD and MPGD are not similar for each mode. With MPGD, for the first mode, it seems that the distribution does not take into account the effect of the conducting plate. For the second mode, the distribution is close to the reaction magnetic field distribution due to the eddy current density. With MPOD, for the first mode, the distribution takes into account the eddy current in a conducting plate. With the second mode, we observe a reaction magnetic flux density due to the eddy current density.

For the global distribution of the magnetic flux density obtained with MPOD and MPGD, the results are close to the one computed with MREF.

\subsection{Computation time}

In terms of computation time, with a length of the time interval of $0.1 \mathrm{~ms}$ and 60 time steps, the MREF requires $5 \mathrm{~min} 7 \mathrm{~s}$ to solve the numerical problem. For MPOD, the computation time is of $1 \mathrm{~min} 50 \mathrm{~s}$, this time takes into account the solution of original model (14) with 5 snapshots and 60 solutions of the reduced model (24). The size of the matrix system to solve is reduced, thus the computation time requires to solve the numerical problem (24) by using an iterative method is small compared to MREF. We should mention that the calculation of the matrices $\boldsymbol{M}_{r}$ and $\boldsymbol{N}_{r}$ and the vector $\boldsymbol{F}_{r}$ which could be cumbersome is not undertaken. In the iterative procedure by using a conjugate gradient method, only matrix-vector products are required, in the algorithm of the method, the product $\boldsymbol{M}_{r} \boldsymbol{X}_{j}$ with $\boldsymbol{X}_{j}$ the vector of the approximated solutions at the $j$-th iteration is calculated by $\boldsymbol{P}^{t} \boldsymbol{M} \boldsymbol{P} \boldsymbol{X}_{j}$. In the linear case, the calculation of the product $\boldsymbol{M}_{r} \boldsymbol{X}_{j}$ without using an explicit expression of $\boldsymbol{M}_{r}$ is not necessarily the most efficient. In the non linear case, it is not possible to determine $\boldsymbol{M}_{r}$ explicitly and the calculation of $\boldsymbol{P}^{t} \boldsymbol{M P} \boldsymbol{X}_{j}$ is required because $\boldsymbol{M}$ is a function of the solution [15][16]. With MPGD, the computation time is of $11 \mathrm{~min}$, this time takes into account the determination of the functions $\boldsymbol{R}_{n}(\boldsymbol{x})$ and $S_{n}(t)$ associated with 15 modes. Each mode requires from 4 to 18 iterations to be determined. Then, 83 numerical solving of (28) and (29) are necessary. In term of computation cost, the numerical solving of (29), which is close to this of MREF, is more important than the solving of (28) due to the number of unknowns in the space domain (mesh) much greater than the number of time steps. We can note that the original PGD method presented in this article can be improved in order to reduce the number of modes with regard to a given precision [6] [7]. To study the influence of the number of time steps on the computation time, a length of the time interval to $0.2 \mathrm{~ms}$ with 120 time steps is considered. The computation time require for MREF, MPOD and MPGD is of $10 \mathrm{~min} 4 \mathrm{~s}, 3 \mathrm{~min} 4 \mathrm{~s}$ and $9 \min 56 \mathrm{~s}$ respectively. As the number of time steps is multiplied by two, so is the computation time for MREF. For MPOD, as the computation cost of the solution of the original model for 5 snapshots is significant compared with this of the reduced model, the computation time is not multiplied by two. For MPGD, the computation time is slightly reduced compared with the first study. In fact, the number of numerical solving of (28) and (29) required to determine the space and time functions is smaller than for the first study (63 times solving). With MPGD, the computation time is less influenced by the number of time steps.

\section{Conclusion}

The Proper Orthogonal Decomposition and the Proper Generalized Decomposition method associated with the vector potential formulation has been developed in order to solve a 3D quasi-static field problem. On the 3D application example, it appears that the accuracy of the solution obtained from both reduction methods is similar compared with this of a fully described model. With the POD model, the reduction of computation time is significant. With the PGD model, the computation time is not proportional to the number of time steps. On the studied example, the POD model requires less computation time and number of modes than the PGD model.

\section{References}

1. J. Lumley, Atmospheric Turbulence and Wave Propagation, $221(1967)$

2. L. Sirovich, Q. Appl.Math., XLV, 561 (1987)

3. Y. Zhai, IEEE Trans. Magn., 43, 1888 (2007)

4. E. Bergische, M. Clémens, IEEE Trans. Magn., 48, 567, (2012)

5. F. Chinesta, A. Ammar, E. Cueto, Archives of Computational Methods in Engineering, 17, 327 (2010)

6. A. Nouy, Computer Methods in Applied Mechanics and Engineering, 199, 1603 (2010)

7. A. Ammar, B. Mokdad, F. chinesta, R. Keunings, Journal of Non-Newtonian Fluid Mechanics, 144, 98 (2007)

8. P. Alotto, M. Guarnieri,F. Moro, A. Stella, IEEE Trans. Magn., 47, 1462, (2011)

9. M. Pineda, F. Chinesta, J. Roger, M. Riera, J. Perez, F. Daim, COMPEL, 29, 919, (2010) 
10. T. Henneron, S. Clénet, Proceeding of COMPUMAG2011

11. T. Henneron, A. Benabou, S. Clénet, IEEE Trans. Magn., $48,1218,(2012)$

12. A. Ammar, F. chinesta, A. Falco, Archives of Computational Methods in Engineering, 17, 473, (2010)

13. F. Chinesta, P. Ladeveze, E. Cueto, Archives of Computational Methods in Engineering, 18, 395, (2011)

14. A. Bossavit, IEEE Trans. Magn., 24, 74 (1988)

15. T. Henneron, S. Clénet, Proceeding of OIPE2012

16. D. Schmidthäusler, S. Schöps, M. Clemens, Proceeding of CEFC2012 